PROCEEDINGS OF THE

AMERICAN MATHEMATICAL SOCIETY

Volume 132, Number 2, Pages 553-561

S 0002-9939(03)07086-

Article electronically published on August 19, 2003

\title{
A CLASSIFICATION OF RAPIDLY GROWING RAMSEY FUNCTIONS
}

\author{
ANDREAS WEIERMANN \\ (Communicated by Carl G. Jockusch, Jr.)
}

\begin{abstract}
Let $f$ be a number-theoretic function. A finite set $X$ of natural numbers is called $f$-large if $\operatorname{card}(X) \geq f(\min (X))$. Let $P H_{f}$ be the Paris Harrington statement where we replace the largeness condition by a corresponding $f$-largeness condition. We classify those functions $f$ for which the statement $\mathrm{PH}_{f}$ is independent of first order (Peano) arithmetic $P A$. If $f$ is a fixed iteration of the binary length function, then $P H_{f}$ is independent. On the other hand $P H_{\log *}$ is provable in $P A$. More precisely let $f_{\alpha}(i):=|i|_{H_{\alpha}^{-1}(i)}$ where | $\left.i\right|_{h}$ denotes the $h$-times iterated binary length of $i$ and $H_{\alpha}^{-1}$ denotes the inverse function of the $\alpha$-th member $H_{\alpha}$ of the Hardy hierarchy. Then $P H_{f_{\alpha}}$ is independent of $P A$ (for $\alpha \leq \varepsilon_{0}$ ) iff $\alpha=\varepsilon_{0}$.
\end{abstract}

\section{INTRODUCTION AND MOTIVATION}

The Peano axioms $P A$ seem to be designed in such a way that every true assertion about the natural numbers (when formulated in a canonical first-order language $L_{P A}$ to reason about natural numbers) should be a logical consequence of these axioms. Nevertheless, in 1931 Gödel showed that there are true metamathematical statements in the language $L_{P A}$ which are not logical consequences of $P A$. Since then mathematicians have been searching for natural and mathematically interesting statements (i.e. statements that are appealing to mathematicians in general and not just to logicians) which are true but not provable from the axiom system $P A$.

In their celebrated 1977 paper 13 Paris and Harrington were able to show that a certain extension of the finite Ramsey theorem is true but not provable in $P A$. Their extension was based on a certain additional largeness requirement for the homogeneous sets which are asserted to exist by Ramsey's theorem.

It is now an obvious mathematical problem to classify this extra largeness condition with respect to its proof-theoretic strength. Moreover it is also natural to ask whether it is possible to give a motivation for this condition in terms of finite combinatorics or even in terms of Ramsey theory. In this article we aim at a complete solution to these problems. In particular we will indicate how the Paris Harrington

Received by the editors February 21, 2002 and, in revised form, July 4, 2002 and September 26, 2002.

2000 Mathematics Subject Classification. Primary 03F30; Secondary 03D20, 03C62, 05D10.

Key words and phrases. Paris Harrington theorem, rapidly growing Ramsey functions, independence results, fast growing hierarchies, Peano arithmetic.

(C)2003 American Mathematical Society 
assertion emerges naturally from the Erdös Rado bound on the Ramsey function 6].

Since these matters are crucial for the paper we discuss these in more detail. For a given set $Y$ let $[Y]^{p}$ be the set of $p$-element subsets of $Y$. Moreover we identify a natural number $k$ with $\{0, \ldots, k-1\}$. Ramsey's theorem (see, for example, 9] for more details) then reads as follows:

$$
(\forall p, k, n)(\exists r)\left(\forall F:[r]^{p} \rightarrow k\right)(\exists Y \subseteq r)\left[\operatorname{card}(Y) \geq n \& F \uparrow[Y]^{p}=\text { constant }\right] .
$$

Moreover the least such $r$ depending on $p, k, n$ is denoted by $r(p, k, n)$. This function is called the Ramsey function. It is known from a classical paper by Erdös Rado [6] that the function $r(n, n, n)$ is bounded by a superexponential function (where the iterations of the exponential function depend linearly on $n$ ).

Let $[n, r]=\{n, n+1, \ldots, r\}$ and $[n, r]^{p}:=[[n, r]]^{p}$. For a given function $f: \mathbb{N} \rightarrow \mathbb{N}$ let $\mathrm{PH}_{f}$ be the assertion

$$
\begin{aligned}
(\forall p, k, n)(\exists r)\left(\forall F:[n, r]^{p} \rightarrow k\right) & (\exists Y \subseteq[n, r]) \\
& {\left[\operatorname{card}(Y) \geq f(\min (Y)) \& F \uparrow[Y]^{p}=\text { constant }\right] . }
\end{aligned}
$$

The least such $r$ depending on $p, k, n$ is denoted by $r_{f}^{*}(p, k, n)$. According to Paris Harrington 1977 [13] the assertion $P H_{i d}$ is true but not provable in $P A$. Equivalently, the function $n \mapsto r_{i d}^{*}(n, n, n)$ grows so fast that its totality cannot be proven in $P A$.

Let $\log ^{*}$ be the inverse function of $n \mapsto r(n, n, n)$, i.e., $\log ^{*}$ is essentially the inverse function of the superexponential function. Then the Erdös Rado bound yields $r_{\log *}^{*}(n, n, n) \leq r(n, n, n)$ as one can verify by a short calculation.

However, one can do much better. Let $|i|_{d}$ be the $d$-th times iterated binary length of $i$ and let $2_{d}(i)$ be the $d$-th times iterated exponential with respect to base 2 and topmost exponent $i$. For a given unbounded and monotonic increasing function $g$ let $g^{-1}$ be its inverse function. We will show later that the Erdös Rado bound yields

$$
r_{f}^{*}(n, n, n) \leq 2_{n+3}(g(n))
$$

where $f(i):=|i|_{g^{-1}(i)}$.

Thus if $g$ is a recursive function whose totality is provable in $P A$, then $P H_{f}$ is provable in $P A$. Moreover (11) shows that for $P H_{f}$ to be an unprovable statement, $f$ has to grow at least as $f(i)=|i|_{g^{-1}(i)}$, where $g$ grows faster than any $P A$ provably recursive function. We will show that this condition is sufficient (at least when $g$ is chosen as $H_{\varepsilon_{0}}$, the $\varepsilon_{0}$-th function from the Hardy hierarchy). In particular it follows that $P H_{i d}$ is independent which gives the original result by Paris and Harrington.

This discussion indicates that the largeness condition (i.e. $\operatorname{card}(Y) \geq \min (Y)$ ) in the Paris Harrington assertion is well motivated, and that its motivation is autonomous in that it does not depend intrinsically on the infinitary version of Ramsey's theorem. A profound discussion of such naturalness conditions for independence results has been provided by Harvey Friedman in some of his contributions to the Foundations of Mathematics project. See, for example, his posting from 05.02.1999 [7].

The paper is largely self-contained. It is in the combinatorial spirit of the Ketonen Solovay analysis of the Paris Harrington theorem [12] and does not use model theoretic methods. For further information on Ramsey theory the reader is referred 
e.g. to [9] and for further information on subrecursive hierarchies the reader may consult, for example, [4, 8], [15].

This paper is based in part on a modification of Buchholz' technical report [3]. According to Buchholz, his paper is based on a manuscript by Loebl and Nešetřil. A final version of the latter manuscript has later been published (cf. 14]). The difference between this paper and Buchholz' paper are the definition of $m(\alpha)$, Lemma 4, assertion (2) of Lemma 5, the third case in Definition 2.2, Case 2.2 in Lemma 6 and the remaining part after the proof of Lemma 6.

\section{Proof of the MAin theorem}

We follow the notations of Buchholz [3]. Thus $d, i, j, k, l, m, n, p, s, t, M, N$ range over natural numbers, $\alpha, \beta, \gamma, \delta, \sigma, \tau, \xi$ range (if not stated otherwise) over ordinals less than $\varepsilon_{0}$ (which by definition is the least ordinal $\xi$ such that $\xi=\omega^{\xi}$ ) and $X, Y, Z$ range over sets.

For formulating the result we fix some terminology and abbreviations. As indicated above, we write $[X]^{p}:=\{Y: Y \subseteq X \& \operatorname{card}(Y)=p\}$ and $[n, N]^{p}:=$ $[\{n, n+1, \ldots, N\}]^{p}$ for $n \leq N$. For each function $F$ with $\operatorname{dom}(F)=[X]^{p}$ we define $Y$ to be $F$-homogeneous iff $Y \subseteq X$ and $\left.\operatorname{card}\left(F[Y]^{p}\right)\right)=1$. We write (in arrow notation)

$$
N \rightarrow_{f}^{*}(n)_{k}^{p}
$$

if $n \leq N$, and for each function $F:[n, N]^{p} \rightarrow k$ there exists an $F$-homogeneous $Y$ such that $\operatorname{card}(Y) \geq f(\min (Y))$. (Note that in particular this yields $\operatorname{card}(Y) \geq$ $f(n)$.) The corresponding Skolem function $r_{f}^{*}$ is defined via $r_{f}^{*}(p, k, n):=\min \{N$ : $\left.N \rightarrow_{f}^{*}(n)_{k}^{p}\right\}$. As usual the well-definedness of $r_{f}^{*}$ follows from the infinite Ramsey theorem.

$\mathrm{PH}_{f}$ is the assertion that the Paris Harrington assertion holds with respect to $f$-largeness. Formally $\mathrm{PH}_{f}$ is the assertion

$$
(\forall p, k, n)(\exists N)\left[N \rightarrow_{f}^{*}(n)_{k}^{p}\right] .
$$

We define $\omega_{0}^{\alpha}:=\alpha, \omega_{n+1}^{\alpha}:=\omega^{\omega_{n}^{\alpha}}$ and $\omega_{n}:=\omega_{n}^{1}$. Further let $h(\alpha):=\min \{n$ : $\left.\alpha<\omega_{n}\right\}$ be the height of $\alpha$.

We write $\alpha={ }_{N F} \omega^{\alpha_{1}} \cdot n_{1}+\cdots+\omega^{\alpha_{t}} \cdot n_{t}$ if $\alpha=\omega^{\alpha_{1}} \cdot n_{1}+\cdots+\omega^{\alpha_{t}} \cdot n_{t}$ and $\alpha>\alpha_{1}>\ldots>\alpha_{t}$ and $n_{1}, \ldots, n_{t}>0$. The existence and uniqueness of such a normal form representation of $\alpha$ is guaranteed by the Cantor normal form from set theory. In this case we write $S_{i}(\alpha):=\omega^{\alpha_{i}} \cdot n_{i}, E_{i}(\alpha):=\alpha_{i}, K_{i}(\alpha):=n_{i}$ for $i \leq t$. For $i>t$ we put $S_{i}(\alpha):=E_{i}(\alpha):=K_{i}(\alpha):=0$. In contrast to Buchholz' paper we define $m(0):=0$,

$$
m(\alpha):=\max \left\{n_{1}, \ldots, n_{t}, m\left(\alpha_{1}\right), \ldots, m\left(\alpha_{t}\right)\right\}
$$

and $p(\alpha):=m(\alpha)+h(\alpha)$. Note that the width $w(\alpha)$ of $\alpha$ does not appear in the definition of $m(\alpha)$ and $p(\alpha)$ where the width $w(\alpha)$ is defined by $w(0):=0$ and $w(\alpha):=\max \left\{t, w\left(\alpha_{1}\right), \ldots, w\left(\alpha_{t}\right)\right\}$ if $\alpha=_{N F} \omega^{\alpha_{1}} \cdot n_{1}+\cdots+\omega^{\alpha_{t}} \cdot n_{t}$. This has for our purposes the crucial effect that the number of elements in $\left\{\alpha<\omega_{h}: p(\alpha) \leq k\right\}$ is not bounded by an elementary recursive function in $k$ and $h$.

If we had defined $r(\alpha):=\max \{m(\alpha), w(\alpha)\}$ and $p^{\prime}(\alpha)=r(\alpha)+h(\alpha)$, then the cardinality of $\left\{\alpha<\omega_{h}: p^{\prime}(\alpha) \leq k\right\}$ would have been bounded by a double 
exponential function 1 This is perfect for proving the Paris Harrington theorem in its original form as shown in [3] but in our case it would cause a problem for proving Theorem 1 since the corresponding set $M(l(i))$, which is defined in the proof of Theorem 1 below, would not contain enough elements.

The standard fundamental sequences are defined as follows. If $\alpha={ }_{N F} \omega^{\alpha_{1}}$. $n_{1}+\cdots+\omega^{\alpha_{t}} \cdot\left(n_{t}+1\right)$, then $\alpha[k]=\omega^{\alpha_{1}} \cdot n_{1}+\cdots+\omega^{\alpha_{t}} \cdot n_{t}+\omega^{\alpha_{t}}[k]$. If $\beta$ is a limit ordinal and $\alpha=\omega^{\beta}$, then $\alpha[k]:=\omega^{\beta[k]}$, and if $\alpha=\omega^{\beta+1}$, then $\alpha[k]=\omega^{\beta} \cdot k$. Further let $\varepsilon_{0}[k]=\omega_{k}$. For technical reasons we put $(\alpha+1)[k]:=\alpha$ and $0[k]:=0$.

Lemma 1. (1) $m(\alpha[k]) \leq \max \{m(\alpha), k\}$.

(2) If $m(\alpha) \leq n$, then $m(\alpha[n]) \leq n$.

Proof. The first assertion follows by induction on $\alpha$. The second assertion is immediate by the first assertion.

For $\alpha \leq \varepsilon_{0}$ we define the Hardy function $H_{\alpha}$ as follows. $H_{0}(n):=n, H_{\alpha+1}(n)=$ $H_{\alpha}(n+1)$ and $H_{\alpha}(n):=H_{\alpha[n]}(n+1)$ if $\alpha$ is a limit. The theory of these functions is developed, for example, in [4, [8], [16].

Lemma 2. Let $\alpha_{n}:=\alpha<\varepsilon_{0}, n>m(\alpha)$ and $\alpha_{i+1}:=\alpha_{i}[i]$ for $i \geq n$. Moreover let $N:=\min \left\{i \geq n: \alpha_{i}=0\right\}$. Then $m\left(\alpha_{i}\right)<i$ for all $i \in\{n, \ldots, N\}$ and $H_{\alpha}(n)=N$.

Proof. Lemma 1 yields $m\left(\alpha_{i}\right)<i$ for $i \geq n$ by induction on $i$. Moreover $H_{\alpha_{i}}(i)=$ $H_{\alpha_{i+1}}(i+1)$ for $N>i \geq n$, and therefore $H_{\alpha}(n)=H_{\alpha_{N}}(N)=H_{0}(N)=N$.

For $\alpha>\beta$ set $d(\alpha, \beta):=\min \left\{i: S_{i}(\alpha)>S_{i}(\beta)\right\}, K(\alpha, \beta)=K_{d(\alpha, \beta)}(\alpha)$, $E(\alpha, \beta)=E_{d(\alpha, \beta)}(\alpha)$.

Lemma 3. If $\alpha>\beta>\gamma, d(\alpha, \beta) \leq d(\beta, \gamma)$ and $K(\alpha, \beta) \leq K(\beta, \gamma)$, then $E(\alpha, \beta)>$ $E(\beta, \gamma)$.

Proof. If $d(\alpha, \beta)=d(\beta, \gamma)=: i$, then $S_{i}(\alpha)>S_{i}(\beta)$ and $K_{i}(\alpha) \leq K_{i}(\beta)$, hence $E_{i}(\alpha)>E_{i}(\beta)$. If $i:=d(\alpha, \beta)<d(\beta, \gamma)=: j$, then $E_{i}(\alpha) \geq E_{i}(\beta)>E_{j}(\beta)$.

Lemma 4. Assume $\beta_{0}>\beta_{1}>\ldots>\beta_{m}$ where $m \geq 2$. Let $\delta_{l}:=E\left(\beta_{l}, \beta_{l+1}\right)$ for $l<m$ and assume $\delta_{0}<\ldots<\delta_{m-1}$. Assume $\beta_{m-1}={ }_{N F} \omega^{\alpha_{1}} \cdot n_{1}+\cdots+\omega^{\alpha_{s}} \cdot n_{s}$ and assume that $\delta_{m-1}=\alpha_{i}$ for some $i \in\{1, \ldots, s\}$. Then there exists a $\tau={ }_{N F}$ $\omega^{\tau_{1}} \cdot p_{1}+\cdots+\omega^{\tau_{t}} \cdot p_{t}$ such that $\beta_{0}={ }_{N F} \omega^{\alpha_{1}} \cdot n_{1}+\cdots+\omega^{\alpha_{i}} \cdot n_{i}+\tau$.

Proof. By induction on $m$. Assume that $\beta_{m-2}={ }_{N F} \omega^{\alpha_{1}} \cdot n_{1}+\cdots+\omega^{\alpha_{i}} \cdot n_{i}+\omega^{\gamma_{1}}$. $q_{1}+\cdots+\omega^{\gamma_{c}} \cdot q_{c}$ where $\delta_{m-2}=\gamma_{j}$ for some $j \in\{1, \ldots, c\}$. Then $\gamma_{l}=\alpha_{l+i}$ and $q_{l}=n_{l+i}$ for $l<j$.

Moreover assume that $\beta_{m-1}={ }_{N F} \omega^{\alpha_{1}} \cdot n_{1}+\cdots+\omega^{\alpha_{i}} \cdot n_{i}+\omega^{\gamma_{1}} \cdot q_{1}+\cdots+\omega^{\gamma_{j-1}}$. $q_{j-1}+\omega^{\xi_{1}} \cdot r_{1}+\cdots+\omega^{\xi_{k}} \cdot r_{k}$ where $\xi_{1}<\delta_{m-2}$.

If $m-2=0$, then put $\tau={ }_{N F} \omega^{\gamma_{1}} \cdot q_{1}+\cdots+\omega^{\gamma_{c}} \cdot q_{c}$ and the assertion follows. If $m-2>0$, then the induction hypothesis yields $\beta_{m-2}={ }_{N F} \omega^{\alpha_{1}} \cdot n_{1}+\cdots+\omega^{\alpha_{i}}$. $n_{i}+\omega^{\gamma_{1}} \cdot q_{1}+\cdots+\omega^{\gamma_{j}} \cdot q_{j}+\tau^{\prime}$ for some suitable $\tau^{\prime}={ }_{N F} \omega^{\tau_{1}^{\prime}} \cdot p_{1}^{\prime}+\cdots+\omega^{\tau_{t^{\prime}}^{\prime}} \cdot p_{t^{\prime}}^{\prime}$. Put $\tau=\omega^{\gamma_{1}} \cdot q_{1}+\cdots+\omega^{\gamma_{j}} \cdot q_{j}+\tau^{\prime}$ and the assertion follows.

Definition 1. If $\beta_{0}>\beta_{1}>\beta_{2}$, then

$\chi\left(\beta_{0}, \beta_{1}, \beta_{2}\right):=\nearrow$ if $d\left(\beta_{1}, \beta_{2}\right)<d\left(\beta_{0}, \beta_{1}\right)$,

$\chi\left(\beta_{0}, \beta_{1}, \beta_{2}\right):=\uparrow$ if $d\left(\beta_{0}, \beta_{1}\right) \leq d\left(\beta_{1}, \beta_{2}\right) \& K\left(\beta_{1}, \beta_{2}\right)<K\left(\beta_{0}, \beta_{1}\right)$,

$\chi\left(\beta_{0}, \beta_{1}, \beta_{2}\right):=\downarrow$ otherwise.

${ }^{1}$ This crucial information has been provided to the author by Philippe Flajolet in a private email. The author is very grateful for this. 
We follow the notation of [3], [14] in choosing the arrows $\nearrow, \uparrow, \downarrow$ for the colours given by $\chi$. Technically we shall only in particular need that these symbols are different from certain natural numbers.

Lemma 5. Let $\beta_{0}>\ldots>\beta_{m}$ with $m \geq 2$ and assume that $c \in\{\nearrow, \uparrow, \downarrow\}$ satisfies

$$
\left\{\chi\left(\beta_{i}, \beta_{i+1}, \beta_{i+2}\right): i \leq m-2\right\}=\{c\} .
$$

(1) If $c=\uparrow$, then $m \leq m\left(\beta_{0}\right)<p\left(\beta_{0}\right)$.

(2) If $c=\nearrow$, then $E\left(\beta_{0}, \beta_{1}\right)<\ldots<E\left(\beta_{m-1}, \beta_{m}\right)$.

(3) If $c=\downarrow$, then $E\left(\beta_{0}, \beta_{1}\right)>\ldots>E\left(\beta_{m-1}, \beta_{m}\right)$.

Proof. 1) Let $k_{i}:=K\left(\beta_{i}, \beta_{i+1}\right)$ for $i<m$. Then $k_{0} \leq m\left(\beta_{0}\right)<p\left(\beta_{0}\right) . c=\uparrow$ yields $k_{0}>\ldots>k_{m-1} \geq 1$, hence $m \leq k_{0} \leq m\left(\beta_{0}\right)<p\left(\beta_{0}\right)$.

2) Let $d_{i}:=d\left(\beta_{i}, \beta_{i+1}\right)$ for $i<m$. If $c=\nearrow$, then $d_{0}>\ldots>d_{m-1} \geq 1$, hence $E\left(\beta_{0}, \beta_{1}\right)<\ldots<E\left(\beta_{m-1}, \beta_{m}\right)$ by the definition of function $d$.

3) follows from Lemma 3 .

We write $k^{(s)}:=k+3+3^{2}+\cdots+3^{s}$.

Definition 2. Definition of $\mathcal{C}_{s}^{k}$ and $\chi_{s}^{k}:\left[\omega_{s}^{k}\right]^{s+1} \rightarrow \mathcal{C}_{s}^{k}$.

(1) $\mathcal{C}_{1}^{k}:=\{0, \ldots, k-1\}, \mathcal{C}_{s+1}^{k}:=\mathcal{C}_{s}^{k} \cup\{\nearrow, \uparrow, \downarrow\}^{s}$. Note that $\operatorname{card}\left(\mathcal{C}_{s}^{k}\right)=k^{(s)}$.

(2) If $\alpha=\omega^{k-1} \cdot m_{0}+\cdots+\omega^{0} \cdot m_{k-1}>\omega^{k-1} \cdot n_{0}+\cdots+\omega^{0} \cdot n_{k-1}=\beta$ where $m_{i}, n_{i} \geq 0$ for $0 \leq i \leq k-1$, then $\chi_{1}^{k}(\alpha, \beta):=\min \left\{i: n_{i}<m_{i}\right\}$. Note that $\chi_{1}^{k}(\alpha, \beta)=1+d(\alpha, \beta)$.

(3) Assume that $s \geq 1, \omega_{s+1}^{k}>\beta_{0}>\ldots>\beta_{s+1}, \delta_{i}:=E\left(\beta_{i}, \beta_{i+1}\right)$ and $c_{i}:=$ $\chi\left(\beta_{i}, \beta_{i+1}, \beta_{i+2}\right)$.

If $c_{0}=\ldots=c_{s-1}=\downarrow$, then $\chi_{s+1}^{k}\left(\beta_{0}, \ldots, \beta_{s+1}\right):=\chi_{s}^{k}\left(\delta_{0}, \ldots, \delta_{s}\right)$.

If $c_{0}=\ldots=c_{s-1}=\nearrow$, then $\chi_{s+1}^{k}\left(\beta_{0}, \ldots, \beta_{s+1}\right):=\chi_{s}^{k}\left(\delta_{s}, \ldots, \delta_{0}\right)$.

In all other cases put $\chi_{s+1}^{k}\left(\beta_{0}, \ldots, \beta_{s+1}\right):=\left(c_{0}, \ldots, c_{s-1}\right)$.

Note that according to Lemma 5 we have $\omega_{s}^{k}>\delta_{0}>\ldots>\delta_{s}$ if $c_{0}=$ $\ldots=c_{s-1}=\downarrow$ and $\omega_{s}^{k}>\delta_{s}>\ldots>\delta_{0}$ if $c_{0}=\ldots=c_{s-1}=\nearrow$.

Lemma 6. Let $1 \leq s, k \& s<m \& c \in \mathcal{C}_{s}^{k} \& \omega_{s}^{k}>\beta_{0}>\ldots>\beta_{m}$. If $\chi_{s}^{k}\left(\beta_{i}, \ldots, \beta_{i+s}\right)=c$ for all $i \leq m-s$, then $m<p\left(\beta_{0}\right)$.

Proof. By induction on $s$.

Base case. $s=1$. Let $\beta_{i}:=\omega^{k-1} \cdot n_{i, 0}+\cdots+\omega^{0} \cdot n_{i, k-1}$. Then $n_{0, c}>\ldots>n_{m, c}$ and thus $m \leq n_{0, c} \leq m\left(\beta_{0}\right)<p\left(\beta_{0}\right)$.

Induction step. Let $1 \leq s, k \& s+1<m \& c \in \mathcal{C}_{s+1}^{k} \& \omega_{s+1}^{k}>\beta_{0}>\ldots>\beta_{m}$ and $\chi_{s+1}^{k}\left(\beta_{i}, \ldots, \beta_{i+s+1}\right)=c$ for all $i \leq m-s-1$.

Case 1. $c \in\{\nearrow, \uparrow, \downarrow\}^{s}$.

Let $c_{i}=\chi\left(\beta_{i}, \beta_{i+1}, \beta_{i+2}\right)$ for $i \leq m-2$. Then for all $i \leq m-s-2$ we have $\left(c_{i}, \ldots, c_{i+s-1}\right)=\chi_{s+1}^{k}\left(\beta_{i}, \ldots, \beta_{i+s+1}\right)=\chi_{s+1}^{k}\left(\beta_{i+1}, \ldots, \beta_{i+s+2}\right)=\left(c_{i+1}, \ldots, c_{i+s}\right)$. Hence $c_{i}=c_{i+1}=\ldots=c_{i+s}$ for all $i \leq m-s-2$ and thus $c_{0}=\ldots=c_{m-2}$. From this together with $\chi_{s+1}^{k}\left(\beta_{0}, \ldots, \beta_{s+1}\right) \in\{\nearrow, \uparrow, \downarrow\}^{s}$ we get $c_{0}=\uparrow$ by part (2) of Definition 2 and then $m<p\left(\beta_{0}\right)$ by assertion (1) of Lemma 5 .

Case 2. $c \in \mathcal{C}_{s}^{k}$. Let $\delta_{i}:=E\left(\beta_{i}, \beta_{i+1}\right)$ for $i \leq m-1$.

Case 2.1. $c_{0}=\ldots=c_{m-2}=\downarrow$.

Then $\delta_{0}>\ldots>\delta_{m-1}$. We have $c=\chi_{s+1}^{k}\left(\beta_{i}, \ldots, \beta_{i+s+1}\right)=\chi_{s}^{k}\left(\delta_{i}, \ldots, \delta_{i+s}\right)$ for all $i \leq m-1-s$. The induction hypothesis yields $m-1<p\left(\delta_{0}\right)$. But $p\left(\delta_{0}\right)<p\left(\beta_{0}\right)$ since $m\left(\delta_{0}\right) \leq m\left(\beta_{0}\right)$ and $h\left(\delta_{0}\right)<h\left(\beta_{0}\right)$. 
Case 2.2. $c_{0}=\ldots=c_{m-2}=\nearrow$.

Then $\delta_{0}<\ldots<\delta_{m-1}$. We have $c=\chi_{s+1}^{k}\left(\beta_{i}, \ldots, \beta_{i+s+1}\right)=\chi_{s}^{k}\left(\delta_{i+s}, \ldots, \delta_{i}\right)$ for all $i \leq m-1-s$. The induction hypothesis yields $m-1<p\left(\delta_{m-1}\right)$. But $p\left(\delta_{m-1}\right)<p\left(\beta_{0}\right)$ since an inspection of the involved Cantor normal form according to Lemma 4 yields that $\delta_{m-1}$ is a subterm of $\beta_{0}$, hence $m\left(\delta_{m-1}\right) \leq m\left(\beta_{0}\right)$ and $h\left(\delta_{m-1}\right)<h\left(\beta_{0}\right)$.

For counting the number of ordinals in certain sets we introduce the slow growing hierarchy $\left(G_{\alpha}\right)_{\alpha<\varepsilon_{0}}$ as follows. $G_{0}(n):=0, G_{\alpha+1}(n)=G_{\alpha}(n)+1$ and $G_{\alpha}(n):=$ $G_{\alpha[n]}(n)$ if $\alpha$ is a limit. Further, for a natural number $l$, let $l_{0}(k):=k$ and $l_{n+1}(k):=$ $l^{l_{n}(k)}$.

Lemma 7. (1) If $\alpha={ }_{N F} \omega^{\alpha_{1}} \cdot n_{1}+\cdots+\omega^{\alpha_{t}} \cdot n_{t}$, then $G_{\alpha}(n)=n^{G_{\alpha_{1}}(n)} \cdot n_{1}+$ $\cdots+n^{G_{\alpha_{t}}(n)} \cdot n_{t}$.

(2) If $m(\beta) \leq n$, then $\operatorname{card}\{\alpha<\beta: m(\alpha) \leq n\} \geq G_{\beta}(n)$.

(3) $\operatorname{card}\left\{\alpha<\omega_{s}^{k}: m(\alpha) \leq l\right\} \geq l_{s}(k)$.

Proof. The first assertion is well known and can be proved by a simple induction on $\alpha$. The second assertion follows by induction on $\beta$ using Lemma 1 The third assertion follows from the first and second assertions.

For a number theoretic function $f$ which converges weakly increasing to infinity we define its inverse function $f^{-1}$ by $f^{-1}(i):=\min \{k: f(k) \geq i\}$.

Theorem 1. (1) Let $f_{d, s}(i):=s+\sqrt[d]{|i|_{s-1}}+2$. Then

$$
r_{f_{d, s}}^{*}\left(s+1, k^{(s)}, 3_{s}(s+k+3)\right) \geq H_{\omega_{s}^{k}}(k-1)
$$

for $s \geq 1, d \geq 2$ and $k \geq d^{2}$.

(2) Let $f_{s}(i):=|i|_{s-2}$. Then

$$
r_{f_{s}}^{*}\left(s+1, k^{(s)}, 3_{s}(s+k+3)\right) \geq H_{\omega_{s}^{k}}(k-1)
$$

for $k \geq 4$ and $s \geq 2$.

(3) Let $h_{\varepsilon_{0}}(i):=|i|_{H_{\varepsilon_{0}}^{-1}(i)}$ and $g(s):=3_{s}(s+4)+2$. Then

$$
r_{h_{\varepsilon_{0}}}^{*}\left(s+1, s^{(s)}, 3_{s}(s \cdot 2+3)\right) \geq H_{\varepsilon_{0}}(s-2)
$$

for $s \geq 4$.

(4) Let $h_{s}(i):=s+H_{\omega_{s+1}}^{-1} \sqrt[(i)]{|i|_{s-1}}+2$. Then

$$
r_{h_{s}}^{*}\left(s+1,\left(d^{2}\right)^{(s)}, 3_{s}\left(s+d^{2}+3\right)\right) \geq H_{\omega_{s+1}}(d)
$$

for $s \geq 1$ and $d \geq 2$.

Proof of the first assertion. Assume $k \geq d^{2}$. Let $\alpha_{k-1}:=\omega_{s}^{k}$ and, for $i \geq k-1$, $\alpha_{i+1}:=\alpha_{i}[i]$. Moreover put $N:=\min \left\{i \geq k-1: \alpha_{i}=0\right\}$. Lemma 2 yields $N=H_{\omega_{s}^{k}}(k)$ and

$$
m\left(\alpha_{i}\right) \leq i
$$

for $k \leq i \leq N$. For any natural number $l$ let

$$
M(l):=\left\{\alpha<\omega_{s}^{k-1}: m(\alpha) \leq l\right\}
$$

and enum $_{M(l)}(i)$ be the $i$-th member of $M(l)$ with respect to the standard ordering of the ordinals less than $\varepsilon_{0}$. Let $n:=3_{s}(s+k+3)$ and put $\beta_{n-1}:=\omega_{s}^{k}$ and

$$
\beta_{i}:=\omega_{s}^{k-1} \cdot \alpha_{l(i)}+\text { enum }_{M(l(i))}\left(2_{s-1}\left(l(i)^{d}\right)-i\right)
$$


for $n \leq i \leq N$ where $l(i)$ is the unique natural number such that $l(i)-1<$ $\sqrt[d]{|i|_{s-1}} \leq l(i)$. Then

$$
k \leq l(i)
$$

for $n \leq i \leq N$. Indeed, this follows from the monotonicity of $l(i)$ and the inequality $k \leq l(n)$ where the latter follows from the choice of $n$, since $k \leq \sqrt[d]{3^{s+k+3}}$ for $k \geq d^{2}$.

Moreover the $\beta_{i}$ are well-defined and $\beta_{i+1}<\beta_{i}<\omega_{s}^{k}$ for $n \leq i<N$ since $\operatorname{card}(M(l(i))) \geq l(i)_{s}(k-1) \geq 2_{s-1}\left(l(i)^{d}\right) \geq i$ by assertion (3) of Lemma 7. We have $m\left(\beta_{i}\right)=\max \left\{m\left(\omega_{s}^{k} \cdot \alpha_{l(i)}\right), l(i)\right\} \leq l(i)<\sqrt[d]{|i|_{s-1}}+1$ by (2) and (3). Hence $p\left(\beta_{i}\right) \leq s+\sqrt[d]{|i|_{s-1}}+2$ for $n \leq i \leq N$. Define $F:[n, N]^{s+1} \rightarrow k^{(s)}$ by

$$
F\left(i_{0}, \ldots, i_{s}\right):=\chi_{s}^{k}\left(\beta_{i_{0}}, \ldots, \beta_{i_{s}}\right) .
$$

Let $Y=\left\{i_{0}, \ldots, i_{m}\right\}<$ be $F$-homogeneous. Then $\left\{\beta_{i_{0}}, \ldots, \beta_{i_{m}}\right\}$ is $\chi_{s}^{k}$-homogeneous, hence Lemma 6 yields $\operatorname{card}(Y)=m+1 \leq \max \left\{p\left(\beta_{i_{0}}\right), s+1\right\}<s+\sqrt[d]{\left|i_{0}\right|_{s-1}}+2 \leq$ $s+\sqrt[d]{|\min (Y)|_{s-1}}+2$. The first assertion follows.

The second assertion follows by putting $d=2$ in the proof of the first assertion and noting that $s+\sqrt[d]{|\min (Y)|_{s-1}}+2 \leq|\min (Y)|_{s-2}$ in this case.

Note that in both cases $N$ is too small for the Ramsey number in question since for the particular partition $F$ there exists a homogeneous set $Y$ which does not satisfy the largeness condition.

For a proof of the third assertion we extend the proof of the (first and) second assertion. Put $M:=H_{\varepsilon_{0}}(s-2)$. Then $H_{\varepsilon_{0}}(s-2)=H_{\omega_{s-2}}(s-1) \leq H_{\omega_{s}^{s}}(s-1)$. Let $k=s$. Then $M \leq N$ where $N:=\min \left\{i \geq k-1: \alpha_{i}=0\right\}$ is defined as in the proof of the first assertion. Let $G$ be the restriction of $F$ to $[n, M]^{s+1}$. If $Z$ is $G$ homogeneous, then $Z$ is $F$ homogeneous and as before $\operatorname{card}(Z) \leq|\min (Z)|_{s-2}$. For $i \leq M$ we have $H_{\varepsilon_{0}}^{-1}(i) \leq H_{\varepsilon_{0}}^{-1}\left(H_{\varepsilon_{0}}(s-2)\right)=s-2$. Thus $h_{\varepsilon_{0}}(i) \geq|i|_{s-2}$ for $n \leq i \leq M$. Since $Z \subseteq[n, M]$ we obtain $|\min (Z)|_{s-2} \leq h_{\varepsilon_{0}}(\min (Z))$. The third assertion follows from the proof of the second assertion.

For a proof of the fourth assertion we extend the proof of the first assertion. Put $M:=H_{\omega_{s+1}}(d)$. Let $k=d^{2}$. Then $H_{\omega_{s+1}}(d)=H_{\omega_{s}^{d}}(d) \leq H_{\omega_{s}^{k}}(k-1)$, hence $M \leq N$. Let $G$ be the restriction of $F$ to $[n, M]^{s+1}$. If $Z$ is $G$ homogeneous, then $Z$ is $F$ homogeneous and as before $\operatorname{card}(Z)<s+\sqrt[d]{|\min (Z)|_{s-1}}+2$. For $i \leq M$ we have $H_{\omega_{s+1}}^{-1}(i) \leq H_{\omega_{s+1}}^{-1}\left(H_{\omega_{s+1}}(d)\right)=d$. Thus $h_{s}(i) \geq s+\sqrt[d]{|i|_{s-1}}+2$ for $n \leq i \leq M$. Since $Z \subseteq[n, M]$ we obtain $s+\sqrt[d]{|\min (Z)|_{s-1}}+2 \leq h_{s}(\min (Z))$.

Corollary 1. Let $f_{\alpha}(i):=|i|_{H_{\alpha}^{-1}(i)}$. Then $P H_{f_{\varepsilon_{0}}}$ is unprovable in $P A$.

Proof. Assume otherwise. Then $r_{f_{\varepsilon_{0}}}^{*}$ would be provably recursive in $P A$. Hence, by a classical result about the provably recursive functions of $P A$ (see, for example, [2], [5], [8], 17] for a proof), there is an $\alpha<\varepsilon_{0}$ such that $r_{f_{\varepsilon_{0}}}^{*}\left(n+1, n^{(n)}, 3_{n}(n \cdot 2+3)\right) \leq$ $H_{\alpha}(n)$ for $n \geq 0$. Assertion (3) of Theorem 1 yields

$$
H_{\varepsilon_{0}}(n-2) \leq r_{\varepsilon_{\varepsilon_{0}}}^{*}\left(n+1, n^{(n)}, 3_{n}(n \cdot 2+3)\right)
$$

for $n \geq 4$. Thus $H_{\varepsilon_{0}}(n+1) \leq H_{\alpha}(n+3)=H_{\alpha+3}(n)$ for $n \geq 1$. This contradicts the fact that $H_{\alpha+3}$ is eventually dominated by $H_{\varepsilon_{0}}$.

Corollary 2. Let $g_{d}(i):=|i|_{d}$. Then, for any fixed $d, P H_{g_{d}}$ is unprovable in PA.

Theorem 2. For $\alpha \leq \varepsilon_{0}$ let $f_{\alpha}(i):=|i|_{H_{\alpha}^{-1}(i)}$. Then $r_{f_{\alpha}}^{*}(n, n, n) \leq 2_{n+3}\left(H_{\alpha}(n+3)\right)$. 
Proof. Let $N:=2_{n+3}\left(H_{\alpha}(n+3)\right)$. Then $N>2_{n+1}\left(n \cdot H_{\alpha}(n+3)\right)+H_{\alpha}(n+3)=: M$. Let $F:[n, N]^{n} \rightarrow n$. Then by restriction $F$ induces a map $G:\left[H_{\alpha}(n+3), M\right]^{n} \rightarrow n$. By Theorem 1 of [6] we find $Y$ homogeneous for $G$ (and hence for $F$ ) such that $\operatorname{card}(Y) \geq H_{\alpha}(n+3)$. Moreover

$$
|\min (Y)|_{H_{\alpha}^{-1}(\min (Y)} \leq|N|_{H_{\alpha}^{-1}\left(H_{\alpha}(n+3)\right)}=H_{\alpha}(n+3) .
$$

Corollary 3. Let $f_{\alpha}(i):=|i|_{H_{\alpha}^{-1}(i)}$. Then $P H_{f_{\alpha}}$ is provable in $P A$ for any $\alpha<\varepsilon_{0}$.

Proof. $H_{\alpha}$ is provably recursive in $P A$ for $\alpha<\varepsilon_{0}$. (See, for example, [2], [8], [16], 17] for a proof.) The assertion follows from Theorem 2 (which can be proved in $P A$ for $\left.\alpha<\varepsilon_{0}\right)$.

Let $\log ^{*}(i):=\min \left\{d:|i|_{d} \leq 2\right\}$.

Corollary 4. $P H_{\log ^{*}}$ is provable in $P A$.

Remarks. An inspection of the proofs above (cf. in particular assertion (4) of Theorem 1) yields corresponding results for the fragments of $P A$. Without proof we mention that similar results also hold for the hydra battle and the Goodstein process. (See, for example, 11], [5] for an introduction into these topics.) Related results for Friedman style miniaturizations can be found in [18]. We conjecture that a refined result also holds for regressive Ramsey functions in the sense of Kanamori and McAloon [10]. (Some preliminary results confirming this conjecture have recently been obtained by Lee and the author.)

\section{ACKNOWLEDGEMENTS}

The author is very grateful to the referee for her or his excellent work. The author thanks the DFG for a Heisenberg grant.

\section{REFERENCES}

1. T. Arai: On the slowly well orderedness of $\varepsilon_{0}$, Mathematical Logic Quarterly 48 (2002), 125-130. MR 2003a:03061

2. B. Blankertz and A. Weiermann: How to characterize provably total functions by the Buchholz operator method. Springer Lecture Notes in Logic 6 (1996), 205-213. MR 98c:03116

3. W. Buchholz: On rapidly growing Ramsey functions. Technical report, München 1991.

4. W. Buchholz, A. Cichon and A. Weiermann: A uniform approach to fundamental sequences and hierarchies. Mathematical Logic Quarterly 40 (1994), 273-286. MR 95g:03035a

5. W. Buchholz and S. Wainer: Provably computable functions and the fast growing hierarchy. Logic and combinatorics, Contemporary Mathematics 65, American Mathematical Society, Providence, RI, 1987, 179-198. MR 88d:03109

6. P. Erdös and R. Rado: Combinatorial theorems on classifications of subsets of a given set. Proceedings of the London Math. Society III Ser. 2., (1952), 417-439. MR 16:455d

7. H. Friedman: "Perfect" statements. Posting the FOM email list from 05.02.1999: http:// www.cs.nyu.edu/pipermail/fom/1999-February/002610.html

8. H. Friedman and M. Sheard: Elementary descent recursion and proof theory. Annals of Pure and Applied Logic 71 (1995), 1-45. MR 96c:03110

9. R. L. Graham, B. L. Rothschild and J. H. Spencer: Ramsey theory. John Wiley and Sons, 1990. MR 90m:05003

10. A. Kanamori and K. McAloon: On Gödel incompleteness and finite combinatorics. Annals of Pure and Applied Logic 33 (1987), 23-41. MR 88i:03095

11. R. Kaye: Models of Peano arithmetic. Oxford Logic Guides 15. The Clarendon Press, Oxford University Press, 1991. MR 92k:03034 
12. J. Ketonen and R. Solovay: Rapidly growing Ramsey functions. Annals of Mathematics (2) 113 (1981), 267-314. MR 84c:03100

13. J. Paris and L. Harrington: A mathematical incompleteness in Peano arithmetic, Handbook of Mathematical Logic (J. Barwise, ed.), North-Holland, Amsterdam, 1977, 1133-1142. MR 56:15351

14. M. Loebl and J. Nešetřil: An unprovable Ramsey-type theorem. Report No. 89598-OR, ISSN 0724-3138 (1989). Published in: Proceedings of the American Mathematical Society 116 (1992), 819-824. MR 93a:03067

15. H. E. Rose: Subrecursion: Functions and Hierarchies. The Clarendon Press, Oxford University Press, 1984. MR 86g:03004

16. S. S. Wainer: A classification of the ordinal recursive functions. Archiv für Mathematische Logik und Grundlagenforschung 13 (1970), 136-153. MR 45:3207

17. A. Weiermann: How to characterize provably total functions by local predicativity, Journal of Symbolic Logic 61 (1996), 52-69. MR 97d:03075

18. A. Weiermann: An application of graphical enumeration to $P A$, Journal of Symbolic Logic 68 , no. 1 (2003), 5-16.

Institut für Mathematische Logik und Grundlagenforschung, Der Westfälischen Wilhelms-Universität Münster, Einsteinstr. 62, D-48149 Münster, Germany

Current address: Department of Mathematics, P.O. Box 80.010, 3508 TA Utrecht, The Nederlands

E-mail address: Andreas.Weiermann@math.uni-muenster.de 